\title{
Control and enhancement of beam quality of broad- area semiconductor devices and arrays by multi-mode interference couplers and two-dimensional gain modulation
}

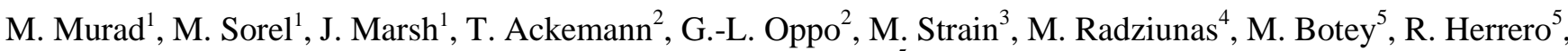 \\ and K. Staliunas ${ }^{5}$ \\ ${ }^{1}$ Department of Electronics \& Electrical Engineering, University of Glasgow, Glasgow G12 8QQ, Scotland, UK \\ ${ }^{2}$ SUPA and Dept. of Physics, University of Strathclyde, Glasgow G4 0NG, Scotland, UK. \\ ("email: thorsten.ackemann@strath.ac.uk) \\ ${ }^{3}$ Institute of Photonics, University of Strathclyde, Glasgow G1 1XQ, Scotland, UK \\ ${ }^{4}$ Weierstrass Institute for Applied Analysis and Stochastics, 10117 Berlin, Germany \\ ${ }^{5}$ Departament de Física i Enginyeria Nuclear, Universitat Politècnica de Catalunya, Terrassa 08222, Spain
}

It is proposed to control the beam quality of broad-area semiconductor devices by two-dimensional structuring enforcing phase coherence across the device. First results based on MMI couplers are presented.

\section{INTRODUCTION}

Diode lasers and amplifiers are a key photonic technology due to their importance as relatively inexpensive, robust, compact and energy efficient high power light sources for many applications. However, there are intrinsic obstacles to achieving simultaneous high-power and a high brightness, since the beam quality of broad-area devices is heavily degraded by spatial hole burning and filamentation dynamics, further exacerbated by spatial inhomogeneities and thermal effects. One approach to control these instabilities is to revert to small-area devices, which run in a fundamental spatial mode, and to couple them in a phase coherent way [1]. However, though next neighbour coupling via evanescent fields results in phase coherent states [1], these are typically anti-phased and not very stable.

The proposal is to control the instabilities by microstructuring on the one hand, but keeping enough global coupling on the other hand to retain coherence across the whole structure. We propose two complementary approaches, both relying on self-imaging effects.

First, starting from an array of small-area lasers, introducing a global on-chip coupling via multi-mode interference couplers (MMI) establishes a stable, global phase coherence across the array and thus provides means to concentrate the output of all lasers on axis.

Second, starting from a broad-area device, we propose to introduce a two-dimensional (simultaneously transverse and longitudinal to the optical axis) modulation of the optical properties of the semiconductor. In particular, we are focusing on the novel approach of a two-dimensional gain modulation, which is considered to be better compatible with semiconductor technology than index modulation required for photonic crystal lasers as it does not require processing very close to the active gain layers. The 2D modulation modifies the spatial dispersion curves and induces an intrinsic angular filtering of the radiation and highly directional on-axis gain, thus strongly improving beam quality and brightness.

We stress that both proposals have the potential for monolithic, on-chip integration, thus maintaining the appeal of semiconductor diodes as compact devices and enabling integration with relative ease into the existing infrastructure.

\section{ARRAYS WITH MMI COUPLERS}

A MMI coupler can be interpreted as a Talbot device with integrated lateral guiding, i.e. it is optimized for self-imaging even with a finite number of elements. The implementation of an array coupled by a $1 \times 4$ coupler is shown in Fig. 1a. Optimal combining of the output from the gain sections into the single mode waveguide is only possible for certain, fixed phase relationships between the array elements.

In the experiment reported here, the emission wavelength was $822 \mathrm{~nm}$, the width of individual emitter $2.5 \mu \mathrm{m}$, array pitch $\mathrm{p}=6 \mu \mathrm{m}$, MMI length $607 \mu \mathrm{m}-630 \mu \mathrm{m}$ and total device length $1.95 \mathrm{~mm}$. The devices were electrically tested with a pulsed current source at $25{ }^{\circ} \mathrm{C}$ using $10 \mu$ s pulses and $1 \mathrm{kHz}$ repetition rate, the output power was measured from the single waveguide facet. The output power can surpass 400 $\mathrm{mW}$ at $2.5 \mathrm{~A}$ injection current.

Far-field patterns and spectra indicate phase-locking in the investigated range up to seven times threshold current. Fig. 1b shows the horizontal far-field pattern with a maximum on axis indicating in-phase locking. Structure and visibility of the far field pattern are as expected for a locked four-element array. 

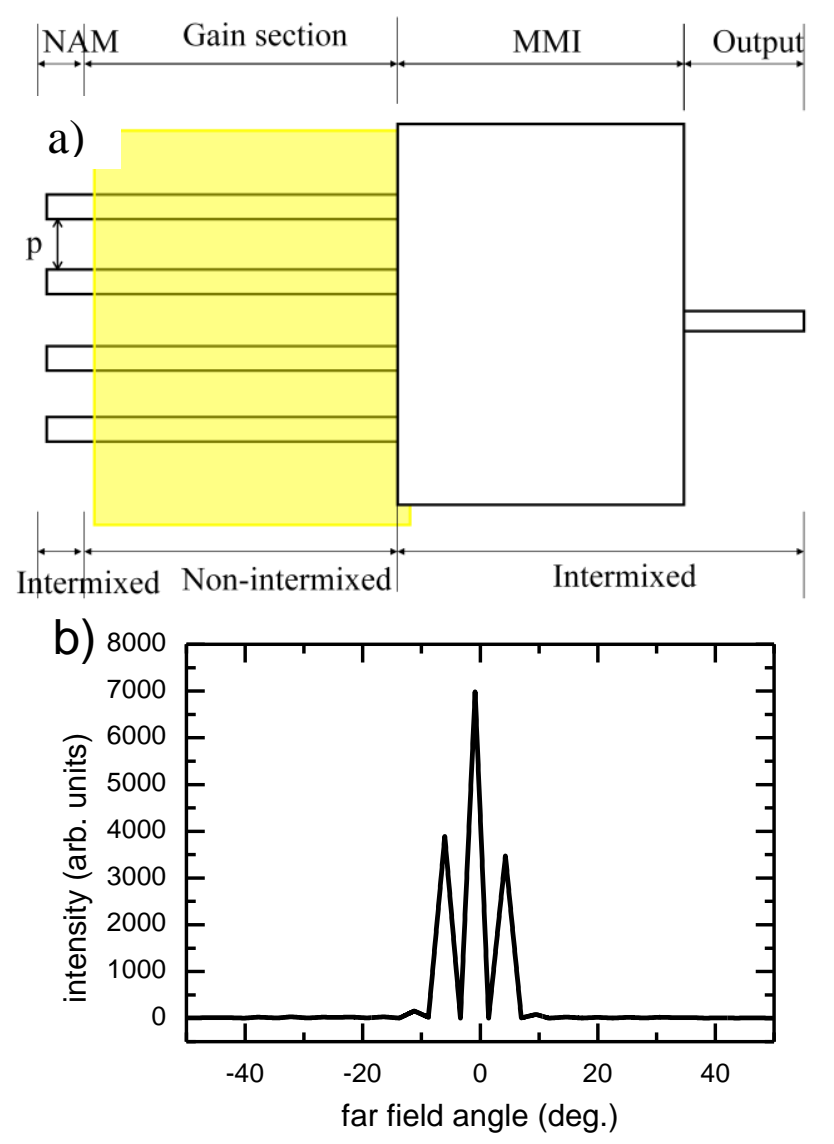

Fig. 1. a) Scheme of a laser array coupled by a $1 \times 4$ MMI coupler to a single fundamental mode waveguide. Passive sections and non-absorbing mirrors (NAM) are generated by quantum-well intermixing. b) Far-field pattern obtained at seven times threshold.

\section{2D GAIN MODULATION}

Photonics structures with a 2D modulation of gain and loss [2] (see Fig. 2a) are much less investigated than photonic crystal (PhC) structures relying on the modulation of refractive index. However, since in semiconductor materials gain and refractive index are intrinsically linked via the linewidth enhancement factor, one needs to consider gain and index modulated media (GIM).

The basic characteristics of beam propagation in a 2D GIM may be described using a simplified model based on a three plane-wave expansion of the Bloch mode [3]. An important adimensional geometry parameter is $\mathrm{Q}$, which depends on the wavenumber of light $\mathrm{k}_{\mathrm{n}}$, the wavenumber of the modulation in the longitudinal direction $\mathrm{q}_{\|}$(z-axis in Fig. 2a) and the wavenumber of the modulation in the transverse direction $\mathrm{q}_{\perp}$ (x-axis in Fig. 2a) as

$$
\mathrm{Q}=2 \mathrm{q}_{\|} \mathrm{k}_{\mathrm{n}} / \mathrm{q}_{\perp}^{2}=2 \Lambda_{\perp}^{2} /\left(\Lambda_{\|} \lambda\right)
$$

The physically interesting range for spatial filtering is around $\mathrm{Q} \approx 1$. Engineering the complex spatial dispersion relations (Fig. 2b) via $\mathrm{Q}$ one can obtain modes with a positive imaginary part of the longitudinal wavevector around $\mathrm{k}_{\perp} \approx 0$ indicating amplification of modes close to the optical axis (black curve, Fig. 2b). Hence the modes with small $\mathrm{k}_{\perp}$ values, propagating at small angles to the optical axis and associated to a smooth beam profile are amplified, while modes at larger angles associated to beam irregularities decay or are less amplified, i.e. the amplified radiation is spatially filtered. The dispersion branch with positive (negative) gain corresponds to the Bloch modes with intensity maxima located at gain (loss) areas of the GIM media. Alternatively one can interpret these two modes in terms of the Talbot effect, one is self-imaging on the gain parts of the structure, one on the loss parts. This opens up the intriguing feature of intrinsic beam shaping.

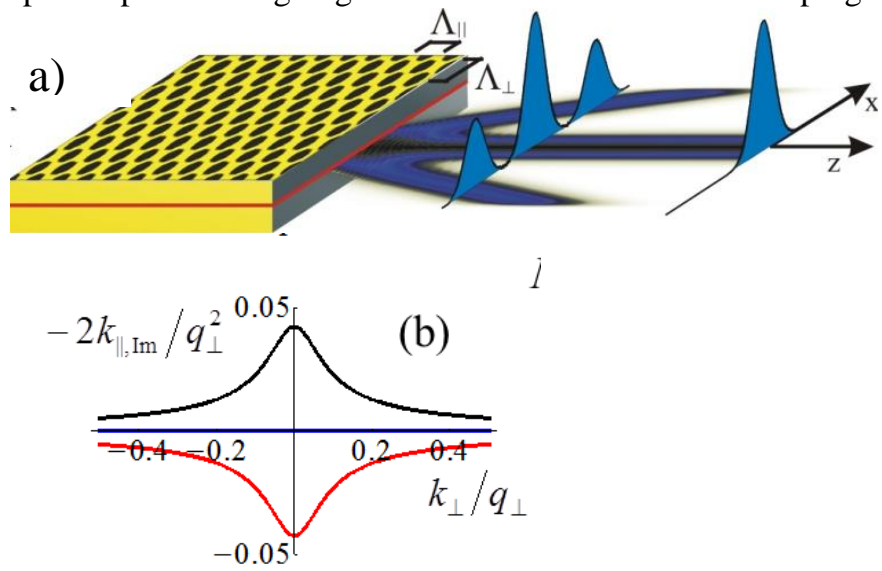

Fig. 2. a) Schematic illustration of a planar edge-emitting semiconductor amplifying structure with a two-dimensional patterning of the gain distribution by structured electrodes providing a spatially periodical modulated pump profile, with transverse and longitudinal periods $\Lambda_{\perp}$ and $\Lambda_{\|}$ respectively.

b) Spatial dispersion curves, showing the imaginary (b) parts of the longitudinal wavevector, $k_{\|}$, for a rhombic gain patterning of the GIM semiconductor with a geometry factor $Q=1$. The real part (not shown) describes the modification (reduction) of diffraction.

\section{CONCLUSION}

Micro-structuring the 2D spatial structure of semiconductor devices seems to be a promising approach to control beam quality of high power semiconductor lasers. The demonstration experiment using MMI couplers constitutes an important proof-of-principle and provides an excellent starting point for further power scaling and far field engineering. Simulations on amplifier models including gain saturation indicate that the $2 \mathrm{D}$ gain modulation approach is feasible beyond the linear limit presented here [4].

\section{REFERENCES}

[1] D. Botez and J.C.Conolly, " High-power phase-locked arrays of indexguided diode lasers," Applied Physics Letters, vol. 43, pp. 1096-1098, 1983.

[2] K. Staliunas, R. Herrero, and R. Vilaseca, "Subdiffraction and spatial filtering due to periodic spatial modulation of the gain-loss profile," Phys. Rev A, vol. 80, pp. 013821, 2009.

[3] R. Herrero, M. Botey, M. Radziunas, and K. Staliunas, "Beam shaping in spatially modulated broad-areasemiconductor amplifiers," Opt. Lett., vol. 37, 5253-5255, 2012.

[4] M. Radziunas, M. Botey, R. Herrero, and K. Staliunas, "Intrinsic beam shaping mechanism in spatially modulated broad area semiconductor amplifiers," Appl. Phys. Lett., vol. 103, 132101, 2013. 